\title{
$X$-ray resonant magnetic scattering study of magnetization reversals in a nanoscale spin-valve array
}

\author{
Dong Ryeol Lee,,${ }^{1 *}$ John W. Freeland, ${ }^{2}$ Yongseong Choi, ${ }^{3}$ George Srajer, ${ }^{2}$ Vitali Metlushko, ${ }^{4}$ and Bojan Ilic ${ }^{5}$ \\ ${ }^{1}$ Pohang Accelerator Laboratory, Pohang University of Science and Technology, Pohang 790-784, Korea \\ ${ }^{2}$ Advanced Photon Source, Argonne National Laboratory, Argonne, Illinois 60439, USA \\ ${ }^{3}$ Materials Science Division, Argonne National Laboratory, Argonne, Illinois 60439, USA \\ ${ }^{4}$ Department of Electrical and Computer Engineering, University of Illinois at Chicago, Chicago, Illinois 60607, USA \\ ${ }^{5}$ Cornell Nanofabrication Facility, Cornell University, Ithaca, New York 14853, USA
}

(Received 9 February 2007; revised manuscript received 15 May 2007; published 19 October 2007)

\begin{abstract}
We present an x-ray resonant magnetic scattering study that uses the periodicity of a patterned array of trilayer $(\mathrm{Co} / \mathrm{Cu} / \mathrm{NiFe})$ elements to determine not only layer-dependent magnetic hysteresis, but, more importantly, to extract the magnetization reversal in different sections of the picture-frame-shaped structure. Spatially resolved and layer-resolved magnetization measurements have revealed that magnetic switching mechanism is very distinct in different regions of the structure and results from a balancing of the shape anisotropy and strong interlayer dipolar coupling. These results demonstrate how spatially averaged measurements are not sufficient to resolve the nature of the reversal mechanism within the structure.
\end{abstract}

DOI: 10.1103/PhysRevB.76.144425

Magnetic multilayered thin-film structures, such as spinvalve structures ${ }^{1}$ and magnetic tunnel junctions, ${ }^{2}$ are the basic elements of the next-generation magnetoelectronic devices. For example, nonvolatile high-density magnetic random access memory ${ }^{3-5}$ (MRAM) and programmable magnetologic gate array ${ }^{6}$ devices. The key issue for their technological application is control of the switching of specific magnetic layers. The basic magnetic elements are composed of two magnetic layers separated by a severalnanometer-thick nonmagnetic spacer or tunneling barrier. In continuous film form, these can be fabricated so that there is little interlayer coupling and the two magnetic layers rotate independently. The magnetic switching mechanisms become more complex when their sizes are in the submicron scale. These dimensions generate significant inter- and intralayer magnetostatic interactions, such as antiparallel interlayer coupling caused by uncompensated poles near the edges, ${ }^{7,8}$ Néel "orange-peel" coupling induced by interface roughness, ${ }^{9}$ and magnetic shape anisotropies due to self-demagnetization. ${ }^{10}$ Moreover, rectangular elements used for devices possess edge domains ${ }^{11}$ and configurational anisotropy. ${ }^{12}$

Interactions between neighboring elements is the major hurdle in increasing the density of MRAM elements. To minimize this effect, topologically designed nanomagnets, such as ring-shaped elements, have been proposed ${ }^{13}$ and experimentally studied, ${ }^{14-16}$ mostly as single-layered elements. Magnetometry measures the total magnetic response and, thus, can only infer the mechanisms of the layer- and domain-dependent magnetic switching. Giant magnetoresistance can be used as a relative measure of differences between the magnetic layers in multilayered devices. ${ }^{16-18}$ However, these are indirect techniques, which can lead to difficulties in determining the uniqueness of the answer. Photoemission electron microscopy has been used to study single multilayered elements in small magnetic fields, ${ }^{19}$ but electron detection makes it difficult to study field-dependent behavior.

In this paper, we show that diffraction of polarized $\mathrm{x}$ rays
PACS number(s): 75.60.Jk, 75.75.+a, 61.10.-i

from a checkerboard array of $\mathrm{Co} / \mathrm{Cu} / \mathrm{NiFe}$ spin-valve nanostructures yields insight into the magnetization reversal process. By using the diffraction amplitudes generated by the periodic structure, the electric field can be localized over specific regions and then magnetic information concerning particular parts of the picture frame can be extracted. The reversal for the section parallel to the magnetic field shows easy-axis behavior with little sign of interlayer coupling, while hard-axis behavior for the section perpendicular to the applied field demonstrates clear signs of strong dipolar coupling between the layers.

An array of $\mathrm{Co}(5 \mathrm{~nm}) / \mathrm{Cu}(3 \mathrm{~nm}) / \mathrm{Ni}_{80} \mathrm{Fe}_{20}(5 \mathrm{~nm}) /$ $\mathrm{Cu}(3 \mathrm{~nm})$ spin-valve picture-frame-shaped squares was fabricated by a combination of e-beam lithography and lift-off techniques. The square picture frames were arranged in a checkerboard-shaped array (see Fig. 1) to minimize interactions between the frames and increase their areal density. $\mathrm{X}$-ray experiments were performed at beamline 4-ID-C of the Advanced Photon Source. ${ }^{20}$ The scattering geometry and the applied magnetic field direction are illustrated in Fig. 1. The diffraction intensities in Fig. 1(a) show a peak spacing corresponding to an array period of $2.15 \mu \mathrm{m}$. Due to the short wavelength and wide slit in the $y$ direction (see inset of Fig. 1), the scattering signal effectively integrates over many orders in the $q_{y}$ direction. ${ }^{21}$ From the best fit of x-ray diffraction intensities, the side length and width of the square frames were 1076 and $225 \mathrm{~nm}$, respectively, which is in agreement with the scanning electron microscope (SEM) micrograph (see inset of Fig. 1). The sample is magnetized parallel to both the sample's surface and the side of the picture frames. To obtain magnetic signals, the difference of the diffraction intensities for left- $\left(I^{+}\right)$and right-handed $\left(I^{-}\right)$circularly polarized incident $\mathrm{x}$ rays was measured. For the scattering geometry used (Fig. 1), the polarization effect of resonant magnetic scattering amplitudes ${ }^{22,23}$ on the difference $\left(I^{+}-I^{-}\right)$contains predominantly the magnetization components projected onto the scattering plane, $M_{x}$ and $M_{z}$, which are the components along the $x$ and $z$ axis, respectively (see Fig. 1). In the following, we concentrate only on $M_{x}$ since 

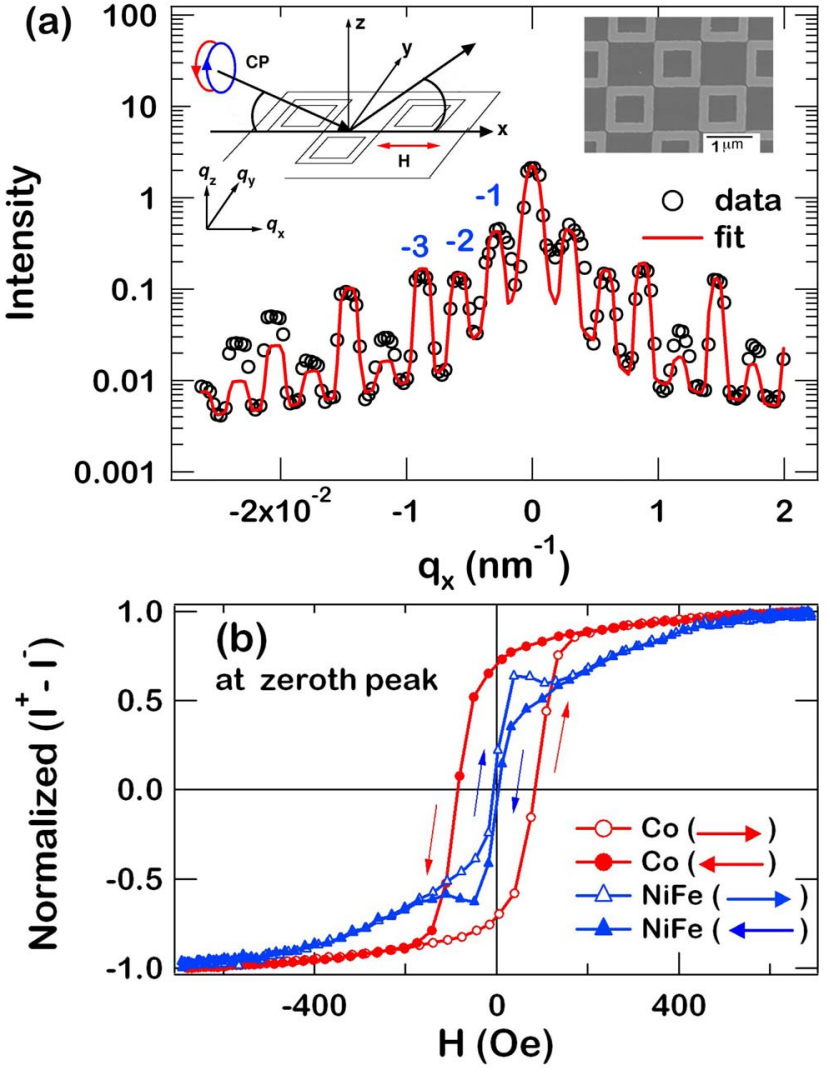

FIG. 1. (Color online) (a) Measured (circles) and calculated (solid lines) diffraction intensities of the sample rocking scan along the $q_{x}$ direction at a fixed detector position $\left(q_{z} \simeq 0.904 \mathrm{~nm}^{-1}\right)$ and the Ni $L_{3}$ edge. Insets: SEM micrograph and schematics of the experimental setup. Circularly polarized $(\mathrm{CP}) \times$ rays were used to probe the sample magnetization along the applied field $H$. (b) The differences $\left(I^{+}-I^{-}\right)$of the specularly reflected intensities at the zeroth order measured at the $\mathrm{Co}$ and $\mathrm{Ni} L_{3}$ edges. The difference intensities are normalized by the saturation amplitudes.

the magnetizations in thin layers lie dominantly in the sample plane $(x-y$ plane $)$. The incident photon energies were tuned to the $\mathrm{Ni}$ and $\mathrm{Co} L_{3}$ edges $(853.5$ and $778.1 \mathrm{eV})$ for the $\mathrm{Ni}_{80} \mathrm{Fe}_{20}$ and $\mathrm{Co}$ layers, respectively, to obtain elementspecific (and, therefore, layer-specific here) magnetic hysteresis loops. Since the specular reflectivity at the zeroth order averages over all parts of the nanostructures, one can directly determine the laterally averaged magnetic hysteresis of each layer, as shown in Fig. 1(b).

To obtain lateral information, we utilized the diffraction amplitudes generated in the plane by the periodicity of the array, which localize the electric field over a certain region of the nanostructure (see inset of Fig. 2). In comparison with the size of the frames, the X-ray wavelength is much smaller $(\sim 1 \mathrm{~nm})$. This, in combination with the wide detector slits in the $y$ direction, leads to a short coherence in the lateral $y$ direction. Consequently, the diffraction peaks can be considered as an incoherent sum of diffracted intensities from a one-dimensional cut of the sample along the $x$ direction. Given the geometry of the sample, there will be two distinct lateral scattering profiles: one cut over the picture frame side parallel to the beam direction (red) and the other cut over the side perpendicular to the beam direction (blue), as shown in the inset of Fig. 2. The electric-field variations at each diffraction peak suggest that the measurements at the first order diffraction peak are most sensitive to the parallel side, while the measurements at the second order peak are most sensitive to the perpendicular side. These selective sensitivities at the two diffraction orders enable us to study the parallel and perpendicular sides of the frames separately. Considering this effect, the dissimilarity between the hysteresis loops at the first and second orders in Fig. 2 reveals that the parallel and perpendicular sides have distinctively different magnetization reversal behaviors.

Next, we have confirmed this simple idea with a full analysis of the data to extract magnetic hysteresis loops of different sections of the nanostructure. As discussed above, the diffraction hysteresis loops are sensitive to laterally inhomogeneous magnetizations due to domain formation and, as a result, show different shapes and amplitudes at different diffraction orders, as shown in Fig. 2. For single layers, this has been reported in diffracted magneto-optical Kerr effect ${ }^{24}$ and spin-resolved neutron scattering ${ }^{25}$ from magnetic hole arrays. Since the hysteresis loops are measured at a limited number of diffraction peaks, we assume that each unit cell of the array is composed of a finite number of magnetic subcells with uniform or averaged magnetizations. While the shapes and sizes of these subcells may be arbitrarily chosen, we limited our choices by energetically possible magnetic domains as well as by geometric configuration and, thus, the total number of mathematically independent subcells can be significantly reduced by the geometrical symmetry. In general, the diffraction hysteresis loops measured at $N$ given diffraction peaks can then provide the magnetization reversals for $N$ magnetic subcells.

The magnetic form factor $f_{m}$ can be given for the $l$ th diffraction peak $\tau_{l}$ and the applied field strength $H$ by

$$
f_{m}\left(\tau_{l}, H\right)=\sum_{n} M_{n}(H) \int_{S_{n}} \exp \left(i \tau_{l} \cdot \mathbf{r}_{x y}\right) d x d y,
$$

where $M_{n}(H)$ is the magnetization for the subcell indexed by $n, \mathbf{r}_{x y}$ is an in-plane position vector, and the integral is carried out over the $n$-indexed subcell area $S_{n}$. Since the difference $\left(I^{+}-I^{-}\right)$is linearly proportional to the magnetic form factor $f_{m}$, X-ray resonant magnetic scattering (XRMS) hysteresis loops measured at $N$ given diffraction peaks can provide $N$ linear equations:

$$
\begin{gathered}
\left(I^{+}-I^{-}\right)_{l=1}(H)=C \sum_{n=1, \ldots, N} F_{l=1, n} M_{n}(H), \\
\left(I^{+}-I^{-}\right)_{l=2}(H)=C \sum_{n=1, \ldots, N} F_{l=2, n} M_{n}(H), \\
\ldots \\
\left(I^{+}-I^{-}\right)_{l=N}(H)=C \sum_{n=1, \ldots, N} F_{l=N, n} M_{n}(H),
\end{gathered}
$$

where $C$ is the constant and $F_{l, n}$ contain both the integral in Eq. (1) and the structural form factor, which is only depen- 


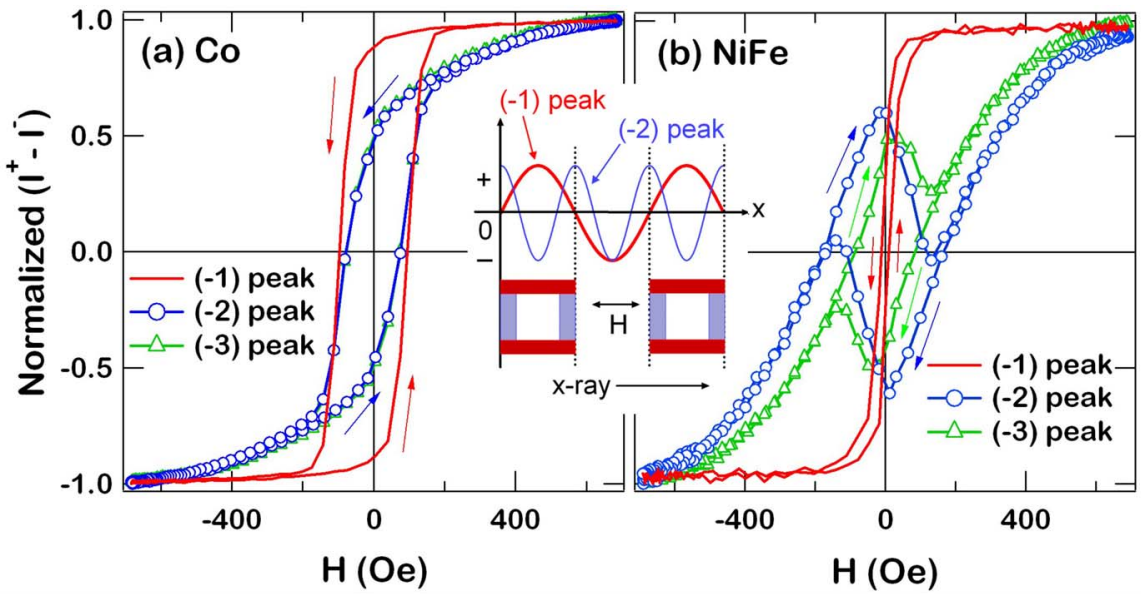

FIG. 2. (Color online) The differences $\left(I^{+}-I^{-}\right)$of the diffraction intensities measured at the different diffraction peaks (indicated by the numbers in Fig. 1) and at the (a) Co and (b) Ni $L_{3}$ edges. Inset: Calculated diffraction amplitudes at the first (red line) and second (blue line) diffraction orders are superimposed over the parallel [red (dark gray)] and perpendicular [blue (light gray)] sides of the picture frame with respect to the beam direction, which is the same as the field direction here.

dent on the diffraction order $l$. Applying linear algebra, the magnetizations for $N$ magnetic subcells can be finally obtained directly from the difference intensities $\left(I^{+}-I^{-}\right)_{l}(H)$ measured at $N$ different diffraction orders by taking the inverse of the $N \times N$ matrix $F_{l, n}$. A detailed discussion can also be found elsewhere. ${ }^{26,27}$

From the hysteresis loops measured at three different orders (Fig. 2), the different magnetization reversals have been directly extracted for three characteristic magnetic subcells [insets of Figs. 3(a)-3(c)]. To confirm the validity of these extracted magnetization loops, an averaged magnetization loop over the unit cell is calculated by summing all extracted loops of three subcells weighted by their area ratio. The calculated sum shown in Fig. 3(d) is in agreement with the measured result (zeroth order) in Fig. 1(b). Now having decomposed the magnetization reversal in different sections of the nanostructure, we can discuss the reversal mechanism in different sections of the picture frame structure.

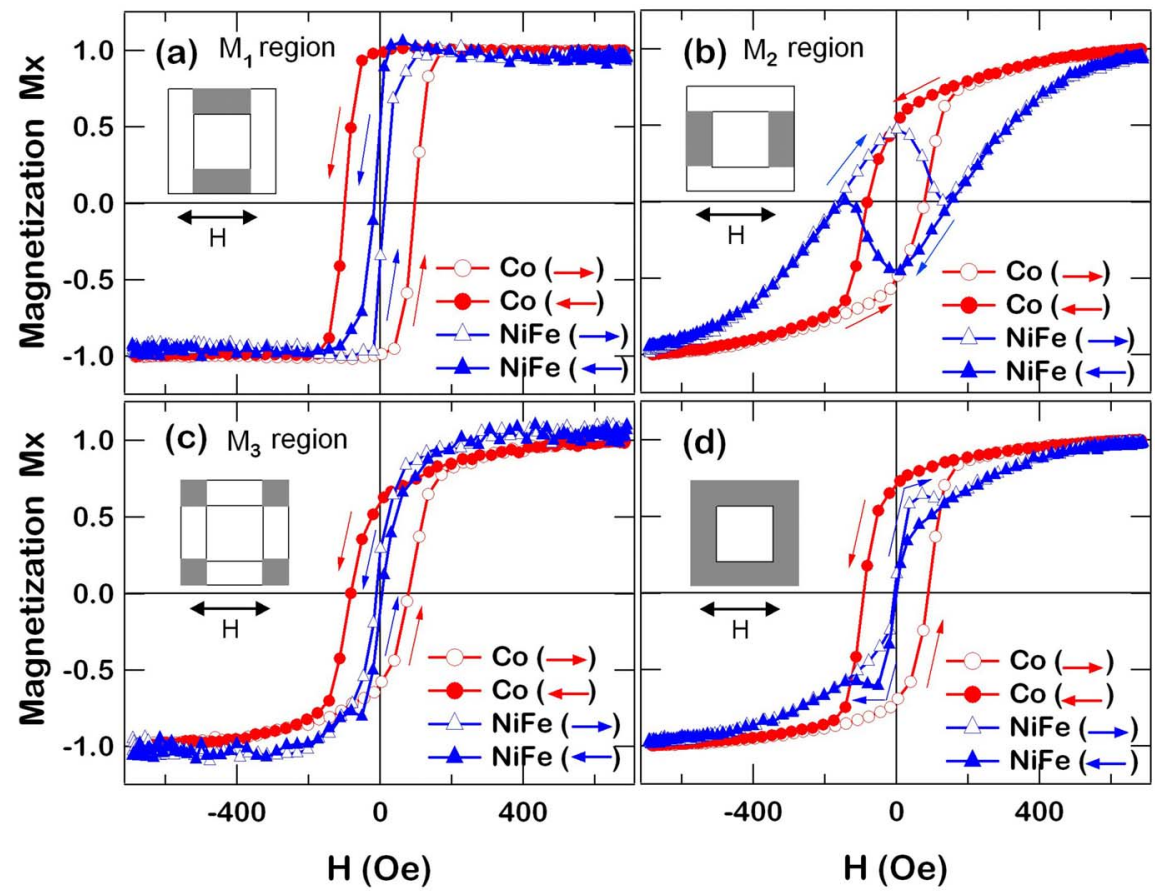

FIG. 3. (Color online) [(a)-(c)] Magnetization reversal behaviors for the three subcells extracted from the hysteresis loops measured at three diffraction orders. The $\mathrm{Co}$ and $\mathrm{NiFe}$ layer magnetization reversal behaviors are shown separately. (d) The calculated magnetization loops obtained by averaging all extracted loops of the three subcells are shown and in agreement with the loops measured by the specular reflectivity in Fig. 1(b). 
First, one sees that in the reversal in the subcell parallel to the field direction $\left[\mathrm{M}_{1}\right.$ region in Fig. 3(a)], both the Co and $\mathrm{NiFe}$ layers show a hysteresis with high remanence and high coercivity due to an easy-axis behavior resulting from shape anisotropy imposed by the geometry of the picture frame. In addition, the $\mathrm{NiFe}$ and Co reversals are quite distinct and free from steps that would indicate significant coupling between the layers. Similar behavior is seen in the corner regions $\left[\mathrm{M}_{3}\right.$ region in Fig. 3(c)]. On the other hand, the loop perpendicular to the field direction $\left[\mathrm{M}_{2}\right.$ region in Fig. 3(b)] shows quite unique shapes. When compared with the Co layer, the inverted loop for the $\mathrm{Ni}_{80} \mathrm{Fe}_{20}$ layer displays in zero field an antiparallel coupling between them (Fig. 4), due to the dipolar field caused by uncompensated magnetic poles at the edges of the Co layer. ${ }^{7,28}$ The insets of Fig. 4 are schematics showing how the dipolar field from the Co layer acts on the $\mathrm{Ni}_{80} \mathrm{Fe}_{20}$ moments through the sides of the picture frames. Since this field favors a closed loop to compensate magnetic poles, the dipolar field is, thus, stronger when increasing the length of the sides perpendicular to the applied field (or resultant magnetization) direction and decreasing the width of the picture frames.

To understand quantitatively this mechanism in the $\mathrm{M}_{2}$ region, we consider the net magnetic field experienced by the $\mathrm{Ni}_{80} \mathrm{Fe}_{20}$ layer. We presume this net field, $H_{\text {net }}^{\mathrm{NiFe}}$, consists of both the applied field $H_{\text {applied }}$ and the demagnetization field $H_{\text {demag }}$ due to the Co layer and can be written as

$$
H_{\text {net }}^{\mathrm{NiFe}}=H_{\text {applied }}+H_{\text {demag }} .
$$

Since $\mathrm{Ni}_{80} \mathrm{Fe}_{20}$ has almost no intrinsic anisotropy, apart from the demagnetization, the $\mathrm{Ni}_{80} \mathrm{Fe}_{20}$ magnetization is determined solely by the shape anisotropy, which prefers the moment under no field to point along the $y$ axis, that is, $M_{x}$ $=0$ in this $\mathrm{M}_{2}$ region. At the field where $M_{x}^{\mathrm{NiFe}}=0$, therefore, the applied field should be cancelled by the demagnetization field from the Co layer. As seen in Fig. 4, there is a particular

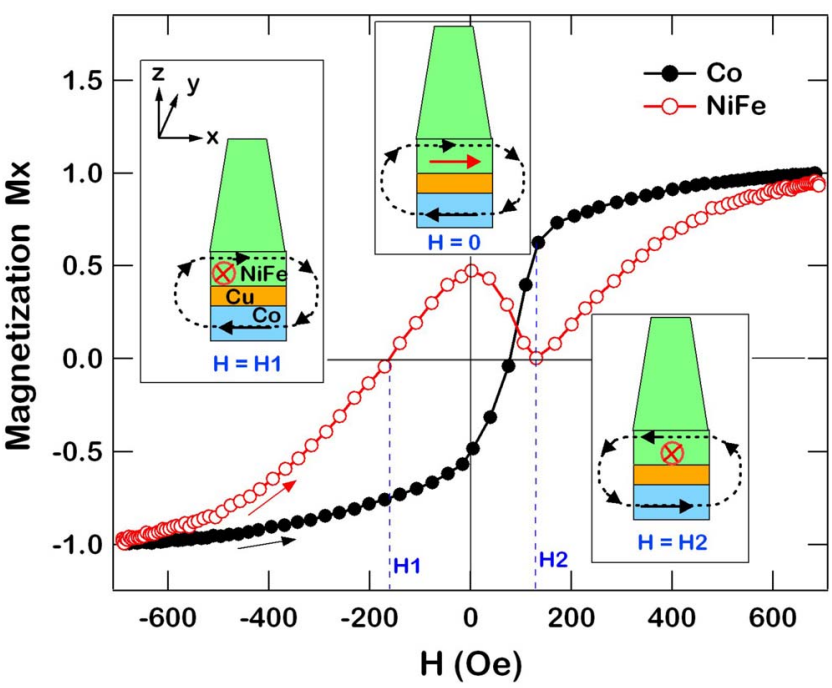

FIG. 4. (Color online) Magnetization reversal mechanism in the $\mathrm{M}_{2}$ region (the sides of the picture frame perpendicular to the field direction). The negative-to-positive curves of the magnetization reversals extracted for the perpendicular sides $\left(\mathrm{M}_{2}\right.$ subcell in Fig. 3) of the picture frames with respect to the field direction are shown for the Co (black line) and $\mathrm{NiFe}$ (red line) layers. The NiFe curve shows an inverted loop. The insets show schematics of how the dipolar field (broken lines with arrows) from the Co layer acts on the NiFe moments through the sides of the picture frames. The solid-line arrows in the insets represent the Co (black arrows) and $\mathrm{NiFe}$ (red arrows) moments, respectively.

field where the $x$ component of the $\mathrm{Ni}_{80} \mathrm{Fe}_{20}$ layer magnetization crosses zero. The inversion of the loop is due to the reduction of the actual applied field due to the demagnetizing field from the Co layer. If the coercivity is zero, $H_{\text {demag }}$ can be directly connected to the Co layer through the following relation:
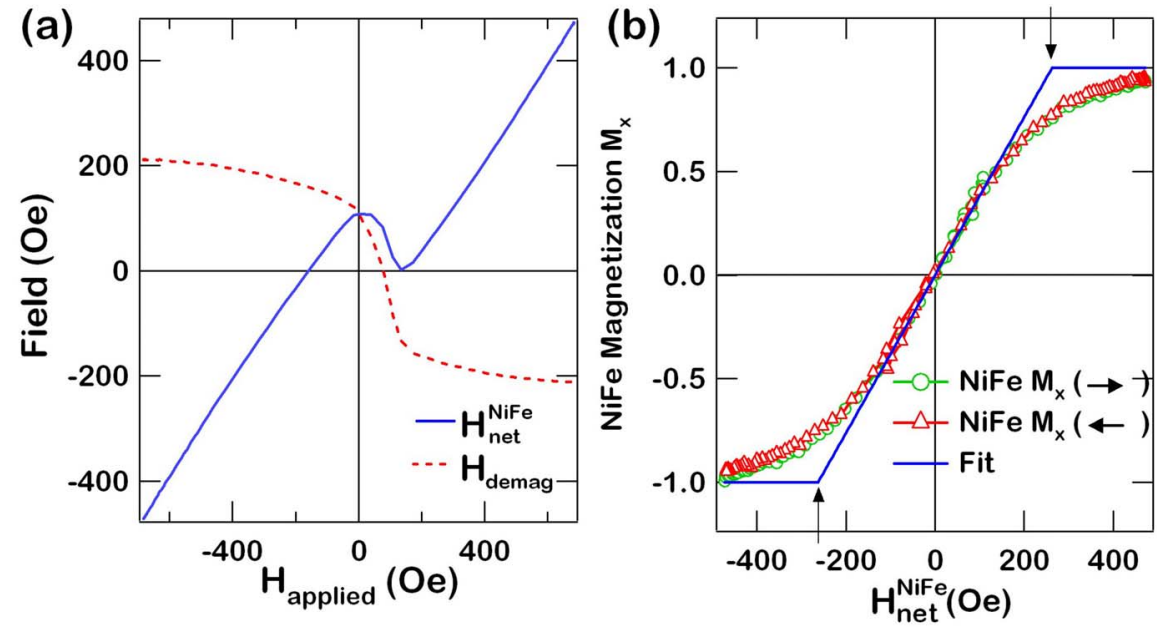

FIG. 5. (Color online) (a) The net field $H_{\text {net }}^{\mathrm{NiFe}}$ (blue line) for the NiFe layer is obtained by subtracting the demagnetization field $H_{\text {demag }}$ (red line), calculated from the magnetization of the Co layer, from the applied field $H_{\text {applied. }}$ (b) The actual magnetization reversal curve under the net magnetic field for the $\mathrm{NiFe}$ layer in the $\mathrm{M}_{2}$ region is shown. This simple S-shaped loop can be fitted by the Stoner-Wohlfarth coherent rotation model (blue line). 


$$
H_{\text {demag }}=-N_{1} M_{x}^{\mathrm{Co}}(H) M_{\mathrm{sat}}^{\mathrm{Co}},
$$

where $N_{1}$ is a constant; $M_{x}^{\mathrm{Co}}$ and $M_{\text {sat }}^{\mathrm{Co}}$ are the $x$ component and the saturation value of the magnetization of the Co layer, respectively; and $H$ represents $H_{\text {applied. This occurs at }}$ $\sim 160$ Oe (Fig. 4), which determines the constant $N_{1}$ of $\sim 0.15$ from Eq. (4) and that $M_{\mathrm{sat}}^{\mathrm{Co}}=1440 \mathrm{emu} / \mathrm{cm}^{3}$. Using the measured constant $N_{1}$, the net field $H_{\text {net }}^{\mathrm{NiFe}}$ and $H_{\text {demag }}$ can be calculated from the above equations and are displayed as a function of the $H_{\text {applied }}$ in Fig. 5(a). Subsequently, the actual $\mathrm{Ni}_{80} \mathrm{Fe}_{20}$ magnetization experienced under the net magnetic field has been reconstructed. The result is a simple $\mathrm{S}$-shaped loop, as shown in Fig. 5(b).

In the classic Stoner-Wohlfarth (SW) coherent rotation model, ${ }^{29}$ this $\mathrm{S}$-shaped loop occurs when the applied field is oriented along the magnetic hard axis, and the saturation field $H_{s}$ has the relationship of $H_{s}=2 K_{u} / M_{\mathrm{sat}}^{\mathrm{NiFe}}$, where $M_{\mathrm{sat}}^{\mathrm{NiFe}}$ is the saturation magnetization of the $\mathrm{Ni}_{80} \mathrm{Fe}_{20}$ layer and $K_{u}$ is the uniaxial shape anisotropy constant. Fitting the slope of the loop near $H=0$ yields the value $H_{s} \approx 250 \mathrm{Oe}$, as indicated by the arrows in Fig. 5(b). From the relationship of $H_{s}$ and $M_{\mathrm{sat}}^{\mathrm{NiFe}}=800 \mathrm{emu} \mathrm{cm}{ }^{-3}, K_{u} \approx 1 \times 10^{5} \mathrm{erg} \mathrm{cm}^{-3}$. This result can be compared with the demagnetization energy $E_{d}$ due to the shape anisotropy, given by $E_{d}=N_{d}\left(M_{\mathrm{sat}}^{\mathrm{NiFe}}\right)^{2} / 2$. Approximating the side of the picture frame as a slender ellipsoid, which is a typical approximation for nanostructures deposited into templates, the demagnetization factor $N_{d}$ in the $\mathrm{M}_{2}$ region of the picture frame can be calculated analytically by $N_{d}$ $=4 \pi t / \mathrm{w}$, where $t$ is the layer thickness and $w$ is the width of the side. ${ }^{30}$ Here, we used $w \approx 40 t$ and the length of the side $l \approx 3 w$ for the calculation of $N_{d}$. For the $\mathrm{M}_{2}$ region of the picture frame used, $N_{d} \approx \pi / 10$ and, subsequently, $E_{d} \approx 1$ $\times 10^{5} \mathrm{erg} \mathrm{cm}^{-3}$, which is in agreement with the measured $K_{u}$ in the SW model.

In summary, we have demonstrated that in-field XRMS based on element-resolved absorption provides a direct measurement of the magnetization hysteresis loops and spatially resolved magnetic states in each individual layer in $\mathrm{Co} / \mathrm{Cu} / \mathrm{NiFe}$ spin valves, and yields additional insight into the magnetization reversal process.

Work at Argonne National Laboratory was supported by U.S. Department of Energy under Contract No. DE-AC0206CH11357. V.M. was supported by the U.S. National Science Foundation (Grant No. ECS-0202780). *drlee@postech.ac.kr

${ }^{1}$ B. Dieny, V. S. Speriosu, S. S. P. Parkin, B. A. Gurney, D. R. Wilhoit, and D. Mauri, Phys. Rev. B 43, 1297 (1991).

${ }^{2}$ J. S. Moodera, L. R. Kinder, T. M. Wong, and R. Meservey, Phys. Rev. Lett. 74, 3273 (1995).

${ }^{3}$ S. S. P. Parkin, K. P. Roche, M. G. Samant, P. M. Rice, R. B. Beyers, R. E. Scheuerlein, E. J. O'Sullivan, S. L. Brown, J. Bucchigano, D. W. Abraham, Y. Lu, M. Rooks, P. L. Trouilloud, R. A. Wanner, and W. J. Gallagher, J. Appl. Phys. 85, 5828 (1999).

${ }^{4}$ S. A. Wolf, D. D. Awschalom, R. A. Buhrman, J. M. Daughton, S. von Molnár, M. L. Roukes, A. Y. Chtchelkanova, and D. M. Treger, Science 294, 1488 (2001).

${ }^{5}$ S. Parkin, X. Jiang, C. Kaiser, A. Panchula, K. Roche, and M. Samant, Proc. IEEE 91, 661 (2003).

${ }^{6}$ A. Ney, C. Pampuch, and H. K. Ploog, Nature (London) 425, 485 (2003).

${ }^{7}$ A. Anguelouch, B. D. Schrag, G. Xiao, Y. Lu, P. L. Trouilloud, R. A. Wanner, W. J. Gallagher, and S. S. P. Parkin, Appl. Phys. Lett. 76, 622 (2000).

${ }^{8}$ C. A. Ross, F. J. Castaño, E. Rodriguez, S. Haratani, B. Vogeli, and H. I. Smith, J. Appl. Phys. 97, 053902 (2005).

${ }^{9}$ B. D. Schrag, A. Anguelouch, S. Ingvarsson, G. Xiao, Y. Lu, P. L. Trouilloud, A. Gupta, R. A. Wanner, W. I. Gallagher, P. M. Rice, and S. S. P. Parkin, Appl. Phys. Lett. 77, 2373 (2000).

${ }^{10}$ R. P. Cowburn, J. Phys. D 33, R1 (2000).

${ }^{11}$ Y. Zheng and J.-G. Zhu, J. Appl. Phys. 81, 5471 (1997).

${ }^{12}$ R. P. Cowburn, A. O. Adeyeye, and M. E. Welland, Phys. Rev. Lett. 81, 5414 (1998).

${ }^{13}$ J.-G. Zhu, Y. Zheng, and G. A. Prinz, J. Appl. Phys. 87, 6668 (2000)

${ }^{14}$ J. Rothman, M. Klaui, L. Lopez-Diaz, C. A. F. Vaz, A. Bleloch, J. A. C. Bland, Z. Cui, and R. Speaks, Phys. Rev. Lett. 86, 1098 (2001).
${ }^{15}$ S. P. Li, D. Peyrade, M. Natali, A. Lebib, Y. Chen, U. Ebels, L. D. Buda, and K. Ounadjela, Phys. Rev. Lett. 86, 1102 (2001).

${ }^{16}$ F. J. Castaño, C. A. Ross, A. Eilez, W. Jung, and C. Frandsen, Phys. Rev. B 69, 144421 (2004).

${ }^{17}$ F. J. Castaño, D. Morecroft, W. Jung, and C. A. Ross, Phys. Rev. Lett. 95, 137201 (2005).

${ }^{18}$ D. Morecroft, F. J. Castaño, W. Jung, J. Feuchtwanger, and C. A. Ross, Appl. Phys. Lett. 88, 172508 (2006).

${ }^{19}$ A. Scholl, J. Stöhr, J. Lüning, J. W. Seo, J. Fompeyrine, H. Siegwart, J.-P. Locquet, F. Nolting, S. Anders, E. E. Fullerton, M. R. Scheinfein, and H. A. Padmore, Science 287, 1014 (2000).

${ }^{20}$ J. W. Freeland, J. C. Lang, G. Srajer, R. Winarski, D. Shu, and D. M. Mills, Rev. Sci. Instrum. 73, 1408 (2001).

${ }^{21}$ D. R. Lee, Y. S. Chu, Y. Choi, J. C. Lang, G. Srajer, S. K. Sinha, V. Metlushko, and B. Ilic, Appl. Phys. Lett. 82, 982 (2003).

${ }^{22}$ J. P. Hannon, G. T. Trammell, M. Blume, and D. Gibbs, Phys. Rev. Lett. 61, 1245 (1988).

${ }^{23}$ J. P. Hill and D. F. McMorrow, Acta Crystallogr., Sect. A: Found. Crystallogr. A52, 236 (1996).

${ }^{24}$ I. Guedes, M. Grimsditch, V. Metlushko, P. Vavassori, R. Camley, B. Ilic, P. Neuzil, and R. Kumar, Phys. Rev. B 66, 014434 (2002).

${ }^{25}$ D. R. Lee, G. Srajer, M. R. Fitzsimmons, V. Metlushko, and S. K. Sinha, Appl. Phys. Lett. 82, 82 (2003).

${ }^{26}$ D. R. Lee, J. W. Freeland, G. Srajer, S. K. Sinha, V. Metlushko, and B. Ilic, arXiv:cond-mat/0309672 (unpublished).

${ }^{27}$ D. R. Lee, J. W. Freeland, G. Srajer, V. Metlushko, and C.-Y. You, J. Appl. Phys. 95, 7016 (2004).

${ }^{28}$ M. Bonfim, G. Ghiringhelli, F. Montaigne, S. Pizzini, N. B. Brookes, F. Petroff, J. Vogel, J. Camarero, and A. Fontaine, Phys. Rev. Lett. 86, 3646 (2001).

${ }^{29}$ E. C. Stoner and E. P. Wohlfarth, Philos. Trans. R. Soc. London, Ser. A 240, 599 (1948); IEEE Trans. Magn. 27, 3475 (1991).

${ }^{30}$ L. Sun, Y. Hao, C.-L. Chien, and P. C. Searson, IBM J. Res. Dev. 49, 79 (2005). 